\title{
Advanced Hybrid Modeling of Hall Thruster Plumes
}

\author{
Tyler D. Huismann ${ }^{*}$ and Iain D. Boyd ${ }^{\dagger}$ \\ University of Michigan, Ann Arbor, MI 48109, USA
}

\begin{abstract}
A hybrid particle-fluid method is applied to model the plume from a $6 \mathrm{~kW}$ Hall thruster operated in the Large Vacuum Test Facility at the University of Michigan. The approach utilizes the direct simulation Monte Carlo method and the Particle-in-Cell method to simulate the collision and plasma dynamics of xenon neutrals and ions. The electrons are modeled as a fluid using conservation equations. A second code is employed to model discharge chamber behavior to provide improved input conditions at the thruster exit for the plume simulation. Simulation accuracy is assessed using experimental data previously recorded.
\end{abstract}

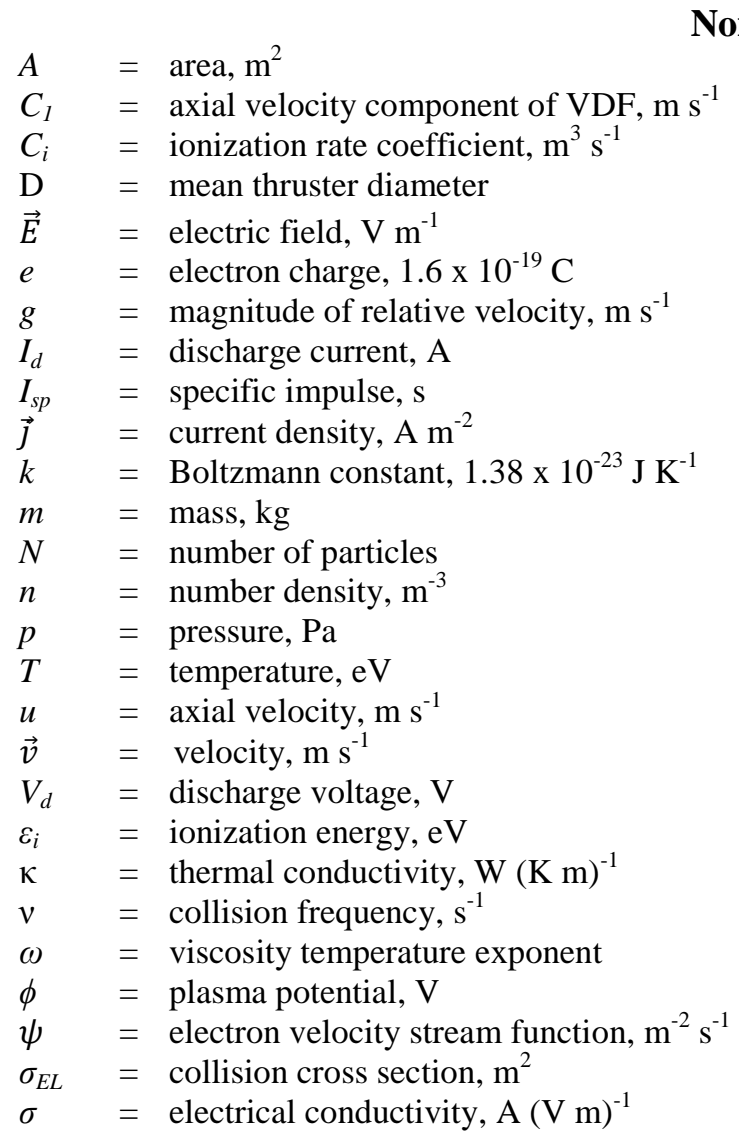

Subscripts
$\mathrm{a}=$ anode
$\mathrm{d}=$ discharge
$\mathrm{e}=$ electron
$\mathrm{i}=$ ion
$\mathrm{TE}=$ thruster exit

\footnotetext{
* Graduate Student Research Assistant, Department of Aerospace Engineering, Student Member, AIAA

${ }^{\dagger}$ Professor, Department of Aerospace Engineering, Associate Fellow, AIAA 


\section{Introduction}

HALL thrusters are an efficient propulsion option for spacecraft, with high specific impulses making them particularly well suited for low thrust missions. A primary concern regarding the use of Hall thrusters is the effect of their plumes on spacecraft integration. Possible spacecraft contamination and communications interference emphasize the importance of accurately analyzing Hall thruster plumes. Two aspects of plume analysis include numerical simulation and ground-based experiments conducted in vacuum chambers. One such facility is the Large Vacuum Test Facility (LVTF) at the University of Michigan's Plasmadynamics and Electric Propulsion Laboratory (PEPL; see Figure 1). It is the focus of the present work to assess plume simulation accuracy through comparisons of simulation results with experimental measurements conducted previously at PEPL.

Hall thruster plume modeling has been reviewed by Boyd ${ }^{1}$ where it was determined that hybrid methods are the most successful. In general, the plume of a Hall thruster consists of neutrals, energetic ions, and electrons. Plume behavior is complicated by the multiple types of collisions involved, such as collisions due to thermal velocity and collisions between neutrals and ions with charge exchange, as well as different physical phenomena, such as selfconsistent electric fields. Furthermore, in ground-based facilities the presence of a background gas must be accounted for. Computational methods are well suited for plume analysis since different physical models can be interchanged to allow for varying degrees of fidelity.

In this paper, a discharge chamber code, HPHall, is applied to model a $6 \mathrm{~kW}$ Hall thruster in order to accurately determine thruster exit conditions. The HPHall predictions are compared to semi-empirical methods that were previously utilized to estimate thruster exit conditions. ${ }^{2}$ A hybrid simulation method is then applied in order to investigate the thruster plume in the LVTF. A direct simulation Monte Carlo (DSMC) method ${ }^{3}$ is used to model collision dynamics, and a Particle-in-Cell (PIC) method ${ }^{4}$ is used to capture electric field effects. A detailed fluidelectron model ${ }^{5}$ is incorporated in place of the standard Boltzmann relation for modeling electrons and is discussed below. Comparisons between the thruster exit conditions are made to determine effects on the calculation of plasma parameters. These comparisons are also extended to experimental measurements previously recorded in the plume, in order to determine the effectiveness of the various methods utilized.

\section{Facility and Thruster}

The LVTF is a $6 \mathrm{~m}$ diameter, $9 \mathrm{~m}$ long cylindrical stainless steel vacuum chamber. The facility is shown in Figure 1. The chamber is pumped by seven CVI TM-1200 re-entrant cryo-pumps which are surrounded by liquid nitrogen $\left(\mathrm{LN}_{2}\right)$ cooled shrouds. The chamber has a total pumping rate of about $240,000 \mathrm{~L} / \mathrm{s}$ for xenon. This resulted in a back-pressure of approximately $1.3 \times 10^{-5}$ torr for nominal operating conditions. The current density measurements utilized in this study were obtained with Faraday cup probes. The near-field current density contours consist of over 64,000 individual measurements of local current density. For further details, see Ref. 6.

The thruster tested in this facility was a $6 \mathrm{~kW}$ Hall thruster. The thruster was operated in the LVTF over a range of operating modes, with the nominal condition being the focus of this work. See Table 1 for the specific operating conditions of this mode. The thruster is a nominal $6 \mathrm{~kW}$ input power thruster designed to produce $100-600 \mathrm{mN}$ of thrust at 1000-3000 s of $I_{s p}$, all stated in Ref. 6. The thruster was equipped with a $\mathrm{LaB}_{6}$ center-mounted hollow cathode that was operated at around $7 \%$ of the anode mass flow rate. For further details of the thruster operation, see the same reference.

\begin{tabular}{|c|c|c|c|}
\hline Operating Condition & $\dot{\boldsymbol{m}}_{\boldsymbol{a}}$ & $\boldsymbol{I}_{\boldsymbol{d}}$ & $\boldsymbol{V}_{\boldsymbol{d}}$ \\
\hline Nominal & $20 \mathrm{mg} / \mathrm{s}$ & $20 \mathrm{~A}$ & $300 \mathrm{~V}$ \\
\hline
\end{tabular}

Table 1: 6kW Hall thruster operating conditions 


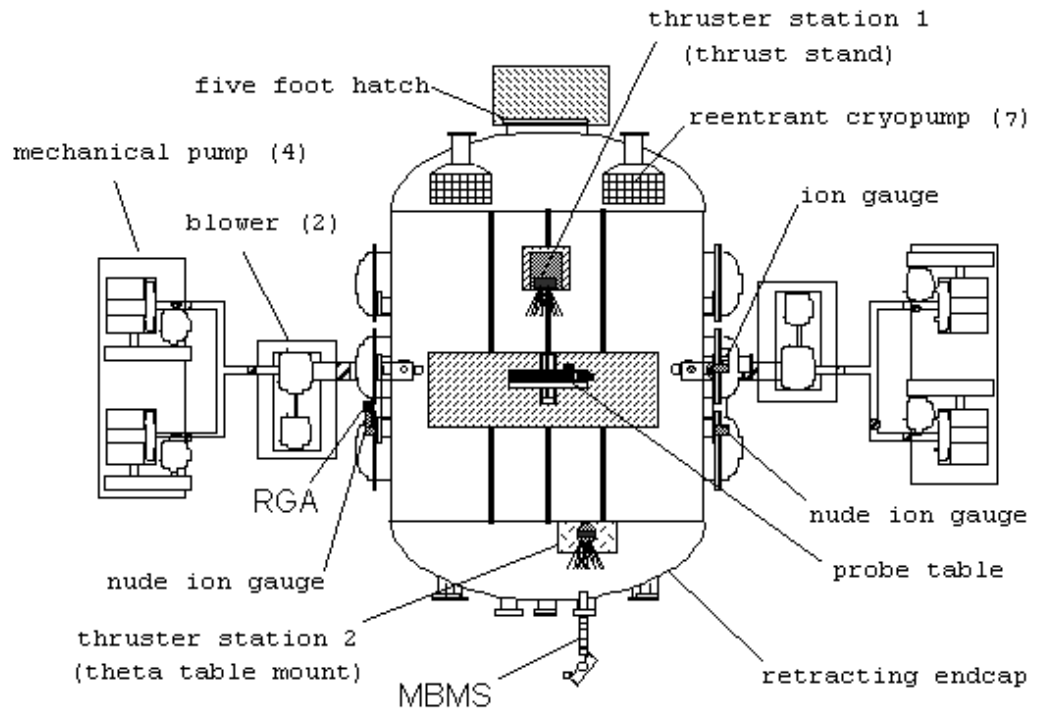

Figure 1: LVTF chamber

\section{III.1 HPHall Methodology}

\section{Numerical Methods}

\section{A. Overview}

For the past decade, the Hall thruster modeling package HPHall has been the focus of much research and analysis. HPHall models the plasma within the thruster discharge chamber and near-field plume, employing both fluid and particle-in-cell (PIC) numerical methods on an axisymmetric grid. ${ }^{7}$ The code has been found to be effective in creating either time-averaged outputs of performance data which could be utilized, for example, in an erosion mode, ${ }^{8}$ or to examine the time-varying nature of the development of the internal plasma structure of a Hall thruster at small timescales. ${ }^{9}$

HPHall's development has been documented by Giuliano and Boyd, ${ }^{10}$ the results of which are summarized here. The HPHall code performs an axisymmetric simulation, commonly referred to as "hybrid-PIC," treating the electrons via fluid approximation equations and treating the heavy species, namely xenon ions and atoms, via a particle-in-cell (PIC) method. The electron equations are solved at a smaller time step, called the electron subcycle, in order to accommodate the fact that the speed of electrons is much faster than that of a xenon atom or ion. This allows for electron fluid equations to be fully converged in between xenon particle updates. Hybrid fluid-particle methods have been shown to be successful in Hall thruster plume studies ${ }^{1}$ and are computationally more efficient than fully kinetic methods. ${ }^{11}$ HPHall, originally created by Fife and Martinez-Sanchez, was later advanced by Gamero-Castano and Katz to include such upgrades as a more robust sheath model and a sputtering yield algorithm for the use as an erosion model. ${ }^{12}$ Further development and corrections were performed by Hofer et al. on the heavy particle modeling, erosion sub-model, and electron mobility physics, continuing the development of HPHall to the present version. ${ }^{8,13-14}$ Through this past work, the code has a favorable history of presenting good agreement with macroscopic properties, such as discharge current and thrust, as well as local properties, such as plasma density, plasma potential, and electron temperature. An example of this time-averaged output of the code can be seen in Figure 2 which is a representation of electron number density at nominal operating conditions, detailed in Table 1. For this type of two-dimensional contour plot, the anode is located on the left side and the near-field plume is located on the right side of the domain.

HPHall has been developed to include the capability to output detailed information of each xenon species at various points in the simulation domain. This capability is utilized in the current study to improve the accuracy of plume simulation input conditions, specifically at the thruster exit. Previous methods used in determining these conditions were semi-empirical. Comparisons between input conditions predicted by these two approaches will be made in Section IV. 


\section{Electron density $\left(\mathrm{m}^{-3}\right)$}

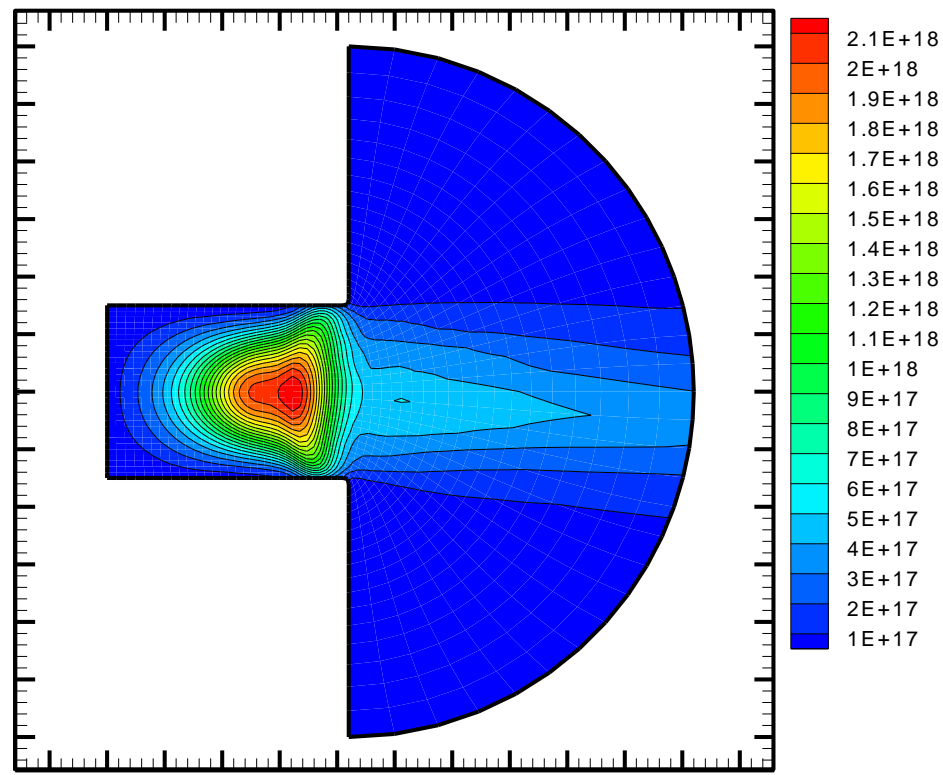

Figure 2: Time-averaged electron number density contours

\section{III.2 DSMC-PIC Methodology}

\section{A. Overview and Collision Dynamics}

The plume simulations use a hybrid DSMC-PIC method. The DSMC module handles collisions. The PIC module is used to move the heavier particles that are influenced by the electric fields present. Finally, the electrons are simulated using a detailed fluid model.

The DSMC module handles collisions between heavier particles $\left(\mathrm{Xe}, \mathrm{Xe}^{+}\right.$, and $\left.\mathrm{Xe}^{++}\right)$, such as neutral-neutral and ion-neutral collisions. The DSMC method uses virtual particles to simulate collisions in rarefied gas flows. The particles represent real ions and neutrals and are grouped in cells whose characteristic lengths are shorter than a mean free path. Pairs of these particles are selected at random and a collision probability is evaluated that is proportional to the product of the relative velocity and collision cross-section. This probability is compared to a random number to determine if the collision occurs. If so, collision dynamics are performed to alter the properties of the colliding particles. Two types of collision dynamics are relevant to Hall thruster plumes: elastic (momentum exchange) collisions and charge exchange collisions (CEX). Elastic collisions involve only exchange of momentum between participating particles. The Hall thruster plume is confined to two different types of momentum exchange collisions, neutral-neutral collisions and neutral-ion collisions. For neutral-neutral collisions, the variable hard sphere model is employed ${ }^{3}$. The cross-section for xenon is:

$$
\sigma_{E L}(X e, X e)=\frac{2.12 \times 10^{-18}}{g^{2 \omega}} m^{2} \quad \text { and } \quad \omega=0.12
$$

where $g$ is the relative velocity and $\omega$ is related to the viscosity temperature exponent for xenon. For neutral-ion elastic interactions, the cross-sections measured by Miller et al. ${ }^{15}$ are used:

$$
\begin{gathered}
\sigma_{E L}\left(X e, X e^{+}\right)=\left(175.26-27.2 \log _{10}(g)\right) \times 10^{-20} \mathrm{~m}^{2} \\
\sigma_{E L}\left(X e, X e^{++}\right)=\left(103.26-17.8 \log _{10}(g)\right) \times 10^{-20} \mathrm{~m}^{2} \\
4 \\
46^{\text {th }} \text { AIAA/ASME/SAE/ASEE Joint Propulsion Conference }
\end{gathered}
$$


Isotropic scattering is assumed for both types of elastic collisions. Charge exchange collisions pertain to the transfer of one or more electrons between an atom and an ion. These cross sections are assumed to follow the same expressions for neutral-ion elastic collisions. However, it is also assumed there is no transfer of momentum accompanying the charge exchange, since it is primarily a long-range interaction.

\section{B. Plasma Dynamics}

The PIC module is used to move the heavier ion particles that are influenced by the electric fields, whereas the lighter electrons are modeled as a fluid. The PIC module determines the charge density at the nodes in the mesh based on the proximity of each particle to the surrounding nodes. The charge density is then used to compute the electric field at each node. This is accomplished either by incorporating the Boltzmann relation or solving for the potential directly using the detailed-fluid model. The potential is then differentiated spatially to obtain the electric fields.

The Boltzmann relation uses several assumptions applied to the electron momentum equation, such as the fluid electron flow being collisionless, isothermal, with no magnetic fields present, and that the electron pressure obeys the ideal gas law, to arrive at the following ${ }^{10}$ :

$$
\phi=\phi_{r e f}+\frac{k T_{r e f}}{e} \ln \left(\frac{n_{e}}{n_{r e f}}\right)
$$

where $\phi$ is the plasma potential, $\phi_{r e f}$ is a reference potential, $k$ is Boltzmann's constant, $e$ is the electron charge, $T_{r e f}$ is the constant electron reference temperature, and $n_{e}$ and $n_{r e f}$ are the local electron number density and reference electron number density, respectively. Reference values are primarily taken at the thruster exit plane. The assumption of quasi-neutrality is employed to obtain the electron number density from the ion number densities.

Most of the strong assumptions made in deriving the Boltzmann relation are questionable in the plume under consideration, especially in the near-field. This is due to strong gradients that could make these approximations inaccurate. Previous work on Hall thruster plume modeling shows the increased fidelity more detailed physics models have over the Boltzmann relation, which in turn provides better agreement with experimental results. ${ }^{2}$ The detailed electron model $^{5}$ increases the modeling fidelity by removing some of the simplifications made with the Boltzmann relation by modeling the electrons with fluid-type conservation equations. The set of Eqs. (5)-(7) is used to increase the level of accuracy by removing some restrictions that the Boltzmann relation assumes, e.g. currentless and isothermal electrons. The relations are manipulated into useful forms for numerical simulation by introducing a stream-function (called the electron velocity potential) for the electron continuity equation and assuming steady state for all the equations. This results in a set of Laplace-type equations with weak source terms, summarized as follows:

$$
\begin{gathered}
\nabla^{2} \psi=n_{e} n_{a} C_{i} \text { where } \nabla \psi=n_{e} \overrightarrow{v_{e}} \\
\nabla(\sigma \nabla \phi)=\frac{k}{e}\left(\sigma \nabla^{2} T_{e}+\sigma T_{e} \nabla^{2}\left(\ln \left(n_{e}\right)\right)+\sigma \nabla\left(\ln \left(n_{e}\right)\right) \cdot \nabla T_{e}+T_{e} \nabla \sigma \cdot \nabla\left(\ln \left(n_{e}\right)\right)+\nabla \sigma \cdot \nabla T_{e}\right) \\
\nabla^{2} T_{e}=-\nabla\left(\ln \left(\kappa_{e}\right)\right) \cdot \nabla T_{e}+\frac{1}{\kappa_{e}}\left(-\vec{\jmath} \cdot \vec{E}+\frac{3}{2} n_{e}\left(\overrightarrow{v_{e}} \cdot \nabla\right) k T_{e}+p_{e} \nabla \cdot \overrightarrow{v_{e}}+3 \frac{m_{e}}{m_{i}} v_{e i} n_{e} k\left(T_{e}-T_{h}\right)+n_{e} n_{a} C_{i} \epsilon_{i}\right)
\end{gathered}
$$

This system is treated in the current study in the same manner as in Refs. 17 and 18, the results of which are summarized as follows. By treating the right-hand side as known in Eqs. (5)-(7), three fundamental plasma parameters are solved for, namely $\vec{v}_{e}, \phi$, and $T_{e}$. This is achieved by expressing the system as a generalized Poisson equation and solving the system with a finite element solver. Derivative calculation is handled by a least squares method, also presented in Ref. 17.

\section{Boundary Conditions}

For computations of a Hall thruster plume in the LVTF, boundary conditions must be specified at the thruster exit, outflow surfaces, chamber centerline, and along the thruster wall, for both the DSMC-PIC method and for the fluid electron model. Figure 3 shows a schematic of the grid utilized in the current study. First, the boundary conditions for the DSMC-PIC method are presented, followed by those for the fluid electron model.

Some of the macroscopic properties of the plasma are required at the thruster exit, namely the number density, velocity components, and temperature of each heavy species in the calculation. Since the particles exit the thruster 


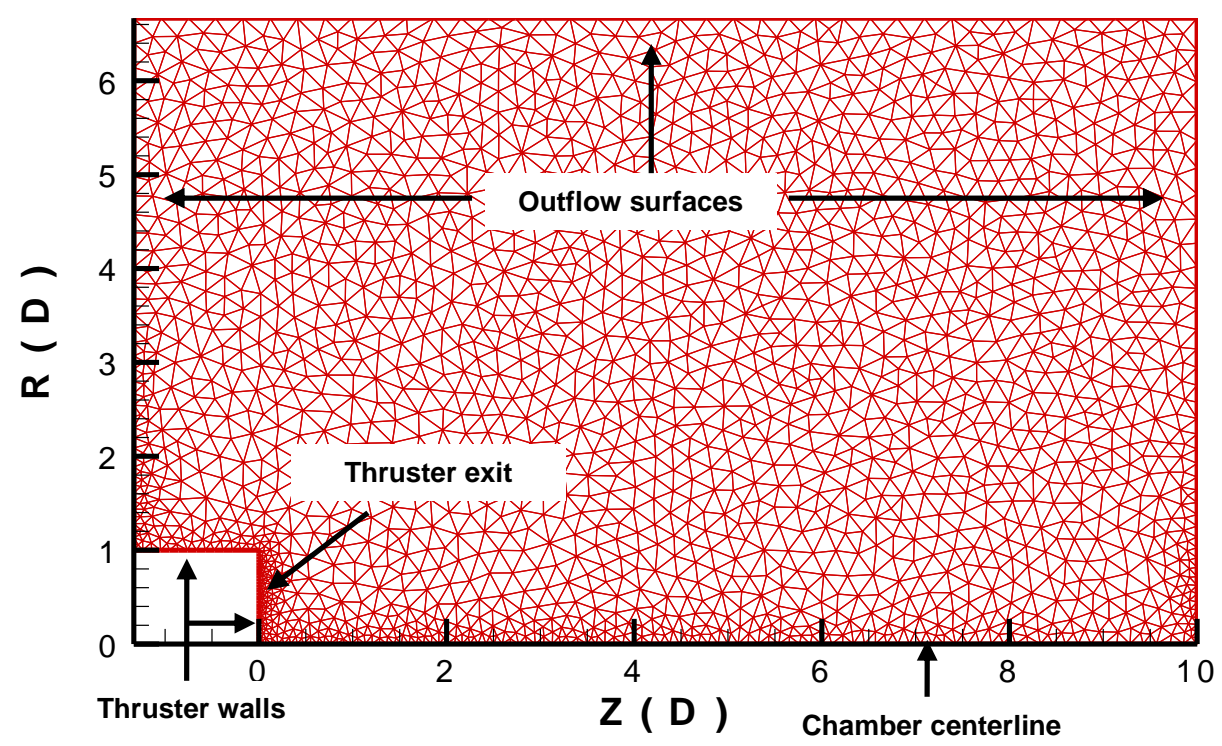

Figure 3: Schematic of axisymmetric, unstructured grid, normalized by mean thruster diameter

with an unknown radial velocity component, this results in a velocity vector that is not parallel to the center-line of the chamber. The angle between the center-line and velocity vector is referred to as the divergence angle, and since it is not known a priori, a sensitivity study is performed that includes a range of assumed values. Particles that exit the thruster plane are assigned a radial velocity component that varies linearly with the distance from the center of the thruster channel. Therefore, a particle exiting the center of the channel will have no radial velocity component, while a particle exiting the channel near its inner or outer wall will have the highest radial velocity component and the angle of the velocity vector with respect to the center-line for that particle will be the divergence angle specified.

The other thruster exit macroscopic properties are determined in general using two methods: first, via a combination of analysis and estimation in order to match experimental operating conditions in the same manner as Ref. 2. The resulting DSMC-PIC input parameters produce thruster exit conditions matching the nominal experimental operating conditions to within 5\% (see Table 1). This has been noted in the same work as a source of uncertainty in comparison with experimental data. Therefore, a second method is employed: conditions are obtained through analysis of HPHall predictions at the thruster exit plane. This approach also results in parameters matching experimental operating conditions to within 5\%. The particle properties predicted by each method are shown in Table 2; see Section IV for further discussion of these predictions.

To determine particle properties at the cathode, the assumption is made that the mass flow consists solely of neutral xenon atoms. This assumption allows for a clear calculation of boundary conditions for the fluid electron model, as injecting xenon ions from the cathode creates a current density which feeds back into determination of the fluid boundary conditions. The neutrals are assumed to have a characteristic temperature of $1300 \mathrm{~K}$ as reported in Boyd $^{19}$. The neutrals injected at the cathode are assumed to have sonic velocity, which, using the reported mass flow rate of $7 \%$ of the anode mass flow rate ${ }^{6}$, thereby determines the number density. The particle properties for species at the cathode are also listed in Table 2 .

There is only one solid surface that is modeled in the present analysis, namely the thruster itself. It is assumed to have a plasma potential of zero, i.e. to be electrically grounded. All ions that collide with the thruster wall are neutralized. For the particles scattered back into the flow field from the thruster wall, diffuse reflection is assumed which is characterized by the surface temperature of $300 \mathrm{~K}$.

The facility back-pressure is modeled through static background particles. Each cell contains a few particles with velocities sampled from a zero-centered Maxwellian velocity distribution function at an assumed temperature of 295 $\mathrm{K}$. These particles participate in collisions with plume particles and change the velocities of other particles, but their positions and velocities do not change. The back-pressure value for these simulations is set to the value near the general operation back pressure, $1.3 \times 10^{-5}$ torr. 


\begin{tabular}{|c|c|c|c|c|c|c|c|c|c|}
\hline \multicolumn{7}{|c|}{ Thruster Particle Properties } & \multicolumn{3}{|c|}{ Cathode Particle Properties } \\
\hline \multirow{2}{*}{ Species } & \multicolumn{2}{|c|}{$n\left(1 / \mathrm{m}^{3}\right)$} & \multicolumn{2}{|c|}{$u(\mathrm{~m} / \mathrm{s})$} & \multicolumn{2}{|c|}{$\mathbf{T}(\mathbf{K})$} & $n\left(1 / \mathrm{m}^{3}\right)$ & $u(\mathrm{~m} / \mathrm{s})$ & $\mathbf{T}(\mathbf{K})$ \\
\hline & $\begin{array}{c}\text { Semi- } \\
\text { empirical }\end{array}$ & HPHall & $\begin{array}{c}\text { Semi- } \\
\text { empirical }\end{array}$ & HPHall & $\begin{array}{c}\text { Semi- } \\
\text { empirical }\end{array}$ & HPHall & \multicolumn{3}{|c|}{$\begin{array}{c}\text { Conditions same for both semi- } \\
\text { empirical and HPHall }\end{array}$} \\
\hline $\mathrm{Xe}$ & $\begin{array}{c}1.77 \mathrm{x} \\
10^{18}\end{array}$ & $\begin{array}{c}2.82 \mathrm{x} \\
10^{16}\end{array}$ & 281 & 410 & 750 & 620 & $\begin{array}{c}1.78 \times \\
10^{20}\end{array}$ & 320 & 1300 \\
\hline $\mathrm{Xe}^{+}$ & $\begin{array}{c}2.00 \mathrm{x} \\
10^{17}\end{array}$ & $\begin{array}{c}3.78 \mathrm{x} \\
10^{17}\end{array}$ & 19600 & 13450 & 23200 & 45000 & 0 & 0 & 0 \\
\hline $\mathrm{Xe}^{++}$ & $\begin{array}{c}5.26 \mathrm{x} \\
10^{16}\end{array}$ & $\begin{array}{c}5.62 \mathrm{x} \\
10^{16}\end{array}$ & 27800 & 17800 & 23200 & 105000 & 0 & 0 & 0 \\
\hline
\end{tabular}

Table 2: Particle properties for DSMC-PIC

The boundary conditions for the detailed-fluid model must be determined in order to solve Eqs. (5) - (7) above. Since each equation is Laplace-like, each one requires specification of either a Dirichlet (direct) value, or a von Neumann (gradient) value. The potential, $\phi$, the electron temperature, $T_{e}$, and the quantity $n_{e} \overrightarrow{v_{e}}$ are specified as either direct or gradient values at each boundary in the simulation. The value to which each is set is specified in Table 3. Each boundary condition is direct except for the following: 1) each boundary condition for the axis of symmetry is a gradient-type condition, 2) the wall of the thruster has a gradient-type condition for the electron temperature, and 3) the outflow regions have a gradient-type condition for $n_{e} v_{e}$. Due to the grounding of the thruster, the plasma potential condition is direct and set to zero for the thruster walls and set to reference values for the thruster exit plane and cathode. The electron temperature is set to characteristic values based on measurements in Ref. 6. The boundary conditions for $n_{e} v_{e}$ are determined using conservation of current as follows:

$$
\begin{gathered}
I_{d}=I_{e}+I_{i} \\
I_{i}=j_{i} * A_{T E} \\
j_{d, \text { cat hode }}=\frac{I_{d}}{A_{\text {cathode }}}
\end{gathered}
$$

where (8) and (9) are used to compute current density at the thruster exit and (10) computes current density at the cathode, from which $n_{e} v_{e}$ is found by dividing by the elementary charge $e$. The sign convention results from electrons flowing out of the cathode, towards the anode. Therefore, the electron velocity stream function must be positive at the cathode and negative at the thruster exit.

\begin{tabular}{|c|c|c|c|c|c|}
\hline \multirow{2}{*}{$\begin{array}{c}\text { Boundary } \\
\text { Condition } \\
\text { Variable }\end{array}$} & \multicolumn{2}{|c|}{ Thruster } & Cathode & $\begin{array}{c}\text { Symmetry-line } \\
\text { (all gradient-type) }\end{array}$ & Outflow \\
\cline { 2 - 3 } & Exit Plane & Body & & $0 \mathrm{~m}^{-3} \mathrm{~s}^{-1}$ \\
\hline$\frac{\partial \Psi}{\partial n}\left(n_{e} v_{e}\right)$ & $-4.64 \times 10^{22} \mathrm{~m}^{-2} \mathrm{~s}^{-1}$ & $0 \mathrm{~m}^{-2} \mathrm{~s}^{-1}$ & $2.85 \times 10^{23} \mathrm{~m}^{-2} \mathrm{~s}^{-1}$ & $0 \mathrm{~m}^{-3} \mathrm{~s}^{-1}$ & $1 \mathrm{~V}$ \\
\hline$\phi$ & $40 \mathrm{~V}$ & $0 \mathrm{~V}$ & $12 \mathrm{~V}$ & $0 \mathrm{~V} \mathrm{~m}^{-1}$ & $1 \mathrm{~V}$ ) \\
\hline$T_{e}$ & $25 \mathrm{eV}$ & $\begin{array}{c}0 \mathrm{eV} \mathrm{m} \\
\text { (gradient) }\end{array}$ & $15 \mathrm{eV}$ & $0 \mathrm{eV} \mathrm{m}^{-1}$ & $1 \mathrm{eV}$ \\
\hline
\end{tabular}

Table 3: Detailed fluid-electron boundary conditions 


\section{Results}

The HPHall simulations are performed as described in Ref. 10: neutral xenon atoms are injected for 20,000 time steps, at which point xenon ions are also injected for an additional 80,000 time steps. The time step size used for the xenon particles is $5 \times 10^{-8}$ seconds, with an electron subcycle time step of $5 \times 10^{-11}$ seconds. The HPHall simulations use approximately 300,000 particles. The plume simulations presented use a total of 500,000 particles at steady state over a domain of 3,925 triangular cells. The plume simulation runs for 300,000 time-steps to reach a steady state and then for another 100,000 time-steps to sample macroscopic data. The time-step size is $1 \times 10^{-6}$ seconds, resulting in a total sampling time of 1 second. Cases are run at a maximum divergence angle of $10^{\circ}$.

\section{A.1 HPHall and Semi-empirical Predictions of Thruster Exit}

Shown in Figures 4-6 are velocity distribution functions (VDF) based on the semi-empirical method and from HPHall for each xenon species. These conditions are then used to construct a Maxwellian VDF which in turn generates injected particles. The semi-empirical method predicts these boundary conditions through estimation based on thruster operating conditions. The method also requires certain assumptions be made, such as specifying $a$ priori the fraction of xenon atoms ionized. The main disadvantage of the semi-empirical method is its imprecision: assumptions must be made about flow conditions with no knowledge regarding the physical situation. This disadvantage was noted in previous work. ${ }^{2}$ As an alternative approach, using HPHall, the xenon species are sampled along the thruster exit, with results shown alongside the semi-empirical results in Figures 4-6. The VDF for each species predicted by HPHall is structurally different in all cases; however, each VDF predicted by HPHall closely matches the structure of a Maxwellian VDF. This congruence allows for the calculation of new boundary conditions for the plume simulations while maintaining the assumption of a Maxwellian VDF at the thruster exit. The new boundary conditions are determined by constructing Maxwellian VDF's that align with those predicted by HPHall; see Figures 7-9.

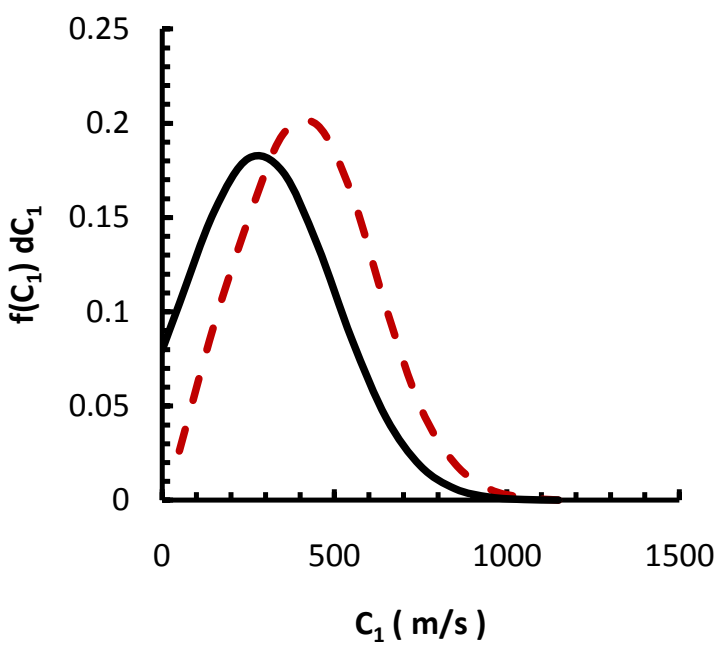

Figure 4: Semi-empirical prediction (solid line) and HPHall prediction (dashed line) for xenon neutral VDF's

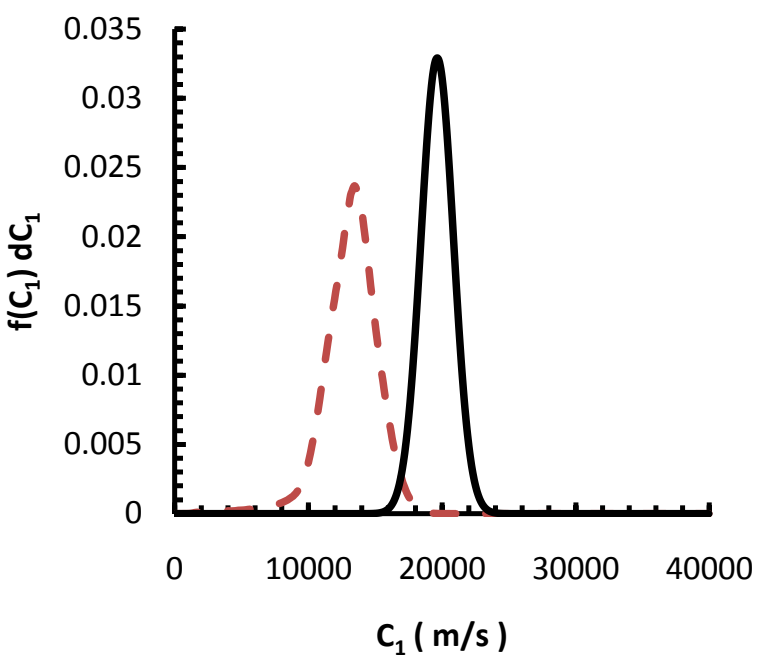

Figure 5: Semi-empirical prediction (solid line) and HPHall prediction (dashed line) for singlecharged xenon ion VDF's 


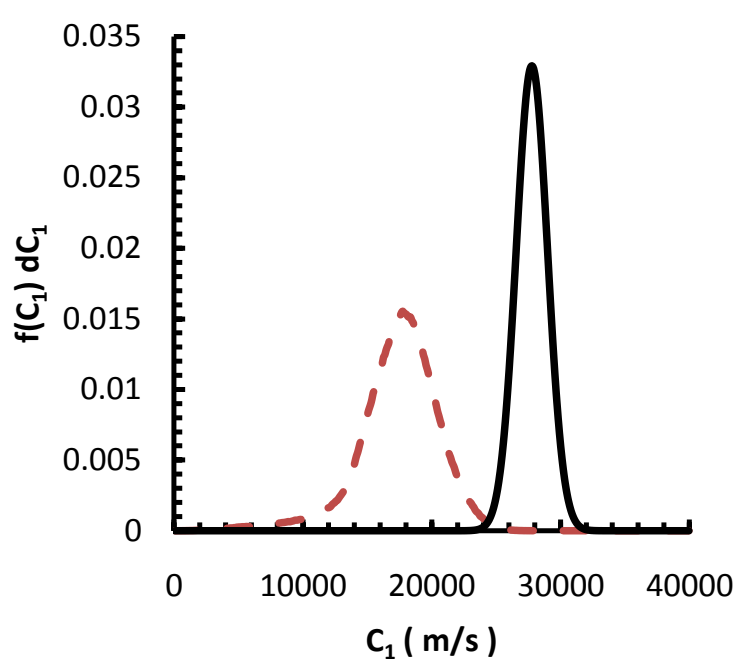

Figure 6: Semi-empirical prediction (solid line) and HPHall prediction (dashed line) for doublecharged xenon ion VDF's



Figure 8: Constructed Maxwellian (solid line) and HPHall prediction (dashed line) for singlecharged xenon ion VDF's

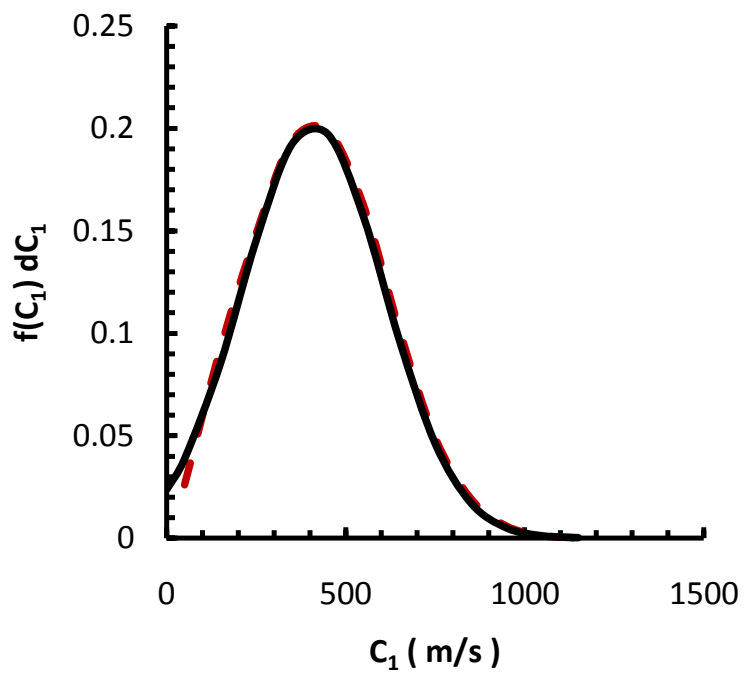

Figure 7: Constructed Maxwellian (solid line) and HPHall prediction (dashed line) for xenon neutral VDF's

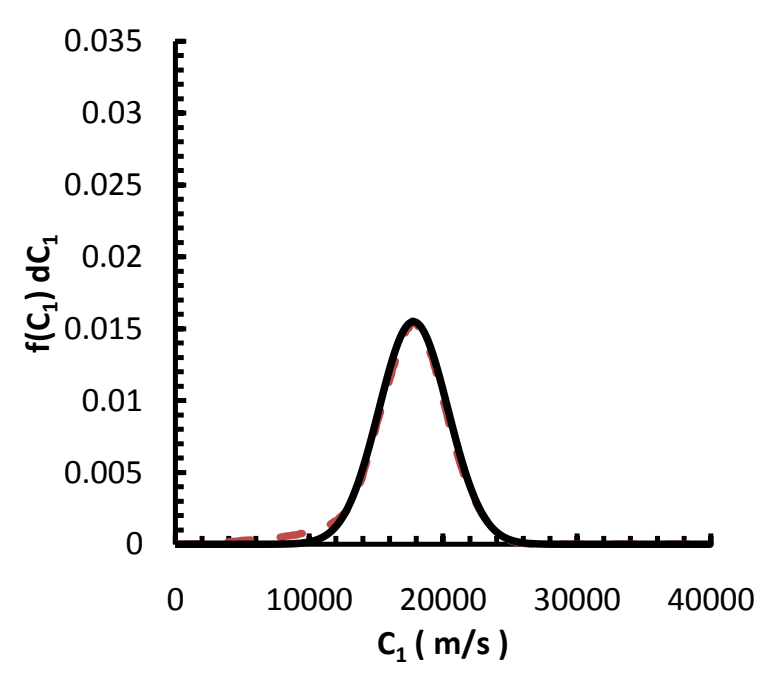

Figure 9: Constructed Maxwellian (solid line) and HPHall prediction (dashed line) for doublecharged xenon ion VDF's 
A comparison of mean velocity, temperature, and number density between the semi-empirical method and the constructed Maxwellian is made in Table 2 above. When compared to the VDF's constructed through HPHall, the semi-empirical method generally over-predicts the mean velocity of ions exiting the thruster, while under-predicting the mean velocity of neutrals. The xenon ion VDF's predicted by the semi-empirical method are also narrower than the constructed VDF's: this results in the HPHall-constructed input conditions specifying a much higher temperature than the temperature assumed by the semi-empirical method. Note that the trend is reversed for the neutral xenon species: the constructed VDF is narrower than the semi-empirical method, resulting in a lower specified temperature for the neutrals.

\section{A.2 Plume Simulations}

There is a wide disparity between the two input conditions, and as a result, the plume simulations that utilize each set of conditions are also disparate. Figures 10 and 11 illustrate the effect of the different thruster conditions on general features of the plume simulations. The differences in the current density contours are mainly attributed to the predicted axial velocity for each method. While the mass flux in each case is the same, the semi-empirical method predicts an axial velocity greater than that predicted by the constructed method by a factor of about 1.5 for both single and double charged xenon species. Because the reference potential is kept constant in these comparisons, the difference in axial velocity is primarily responsible for the longer beam-like plume for the semiempirical conditions, as seen in Figure 10. The effect of these changes on the electron temperature can be seen in Figure 11: the constructed conditions result in a higher peak electron temperature near the thruster exit.

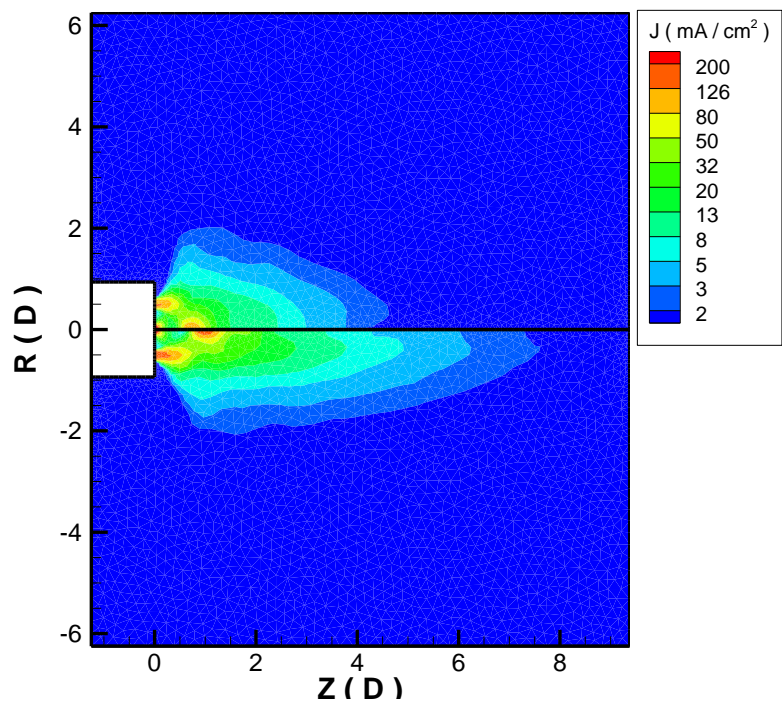

Figure 10: Current density comparison, constructed input condition (top) and semiempirical input condition (bottom)

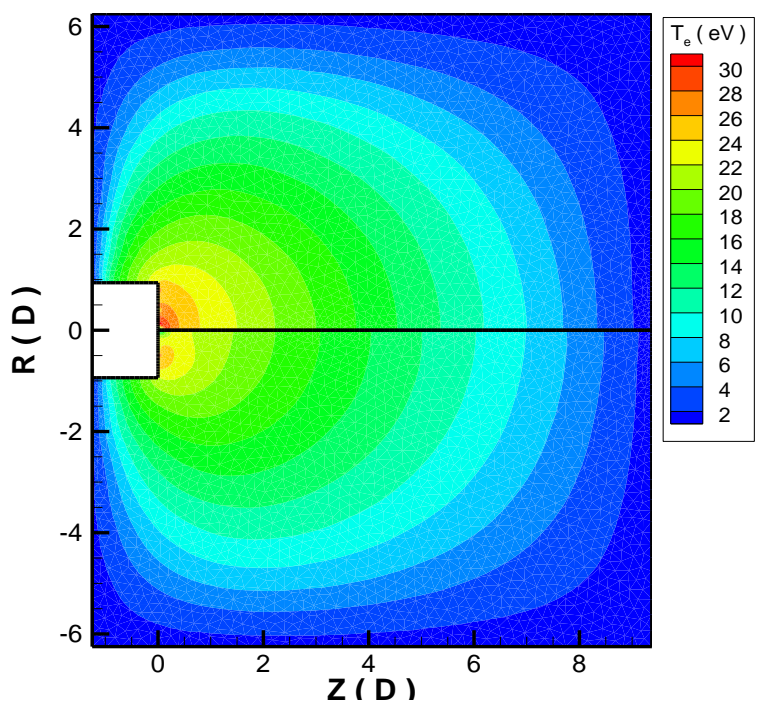

Figure 11: Electron temperature comparison, constructed input condition (top) and semiempirical input condition (bottom)

\section{B. Experimental Comparison}

Data measured in the plume of the $6 \mathrm{~kW}$ thruster are reported in Ref. 6, where data acquisition methods are also described. All measurements are taken at the nominal thruster operating condition. Model assessment is performed through comparison with data measured by a nude Faraday cup probe. For the operating condition under consideration, over 64,000 individual ion current density measurements were taken in the near-field region. The probe did not incorporate a guard ring in this case, as the local Debye length made this impractical. Measurement 
uncertainty is well documented in Ref. 6. Therein uncertainties due to probe collection area, sheath expansion, and cosine losses are reported and conservative estimates suggest uncertainties of $\pm 10 \%$ for ion current density measurements.

Shown below in Figures 12 and 13 are contour plots comparing experimental measurements and various simulations. Contour plots are used to show the effect of method used to determine thruster exit conditions. The main structural similarities between experimental measurements and simulations are in and around the highly collimated plume at the thruster exit. Throughout the first thruster diameter, the main thruster plume remains beamlike regardless of which method is used to determine thruster boundary conditions. Each method also predicts coalescence of the main beam near the chamber centerline. However, the semi-empirical method over-predicts the peak current density in the main ion beam by $33.79 \%$, whereas the constructed boundary conditions come much closer to this peak, differing by only $-1.26 \%$. This discrepancy is highlighted in Figure 14, which shows current density variation along the discharge chamber centerline. Figure 14 illustrates the advantage of using HPHall to construct boundary conditions that are a closer approximation to the physical state at the thruster exit than the approximation using the semi-empirical method.

Figures 15-18 illustrate differences in plume structure by examining radial variation of ion current density at different locations downstream of the thruster exit; Figure 19 compares far-field current density measurements with predictions via both methods. Figures 15 and 16 compare current density measurements and predictions via the semi-empirical method, whereas Figures 17 and 18 compare the same measurements with predictions via the constructed method. Figure 19 compares angularly resolved current density measurements taken on a 1-m circle extending $\pm 180^{\circ}$ from the thruster centerline.

As seen in Figures 15-18, there are two peaks in current density immediately downstream of the thruster exit $(0.1$ D), due to the mass flows from both the thruster and the cathode. Both simulations and measurements show that further downstream the two peaks begin to coalesce, with measurements having almost no sign of a second peak by 1.2 D downstream of the thruster exit. Both simulations predict this coalescence occurring in a shorter downstream distance than measurements: by around $0.5 \mathrm{D}$ downstream of the thruster exit, both simulations predict a single peak current density.

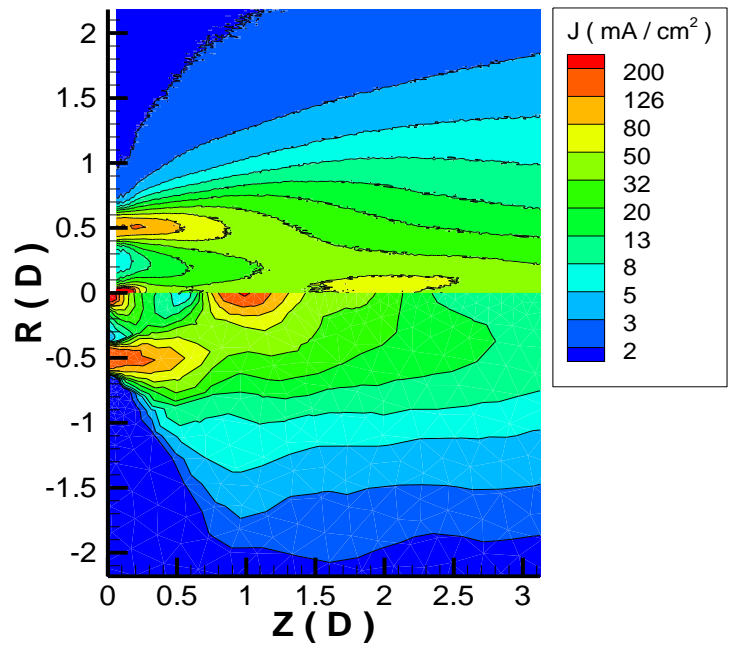

Figure 12: Current density comparison, measured data (top) and semi-empirical input condition (bottom)

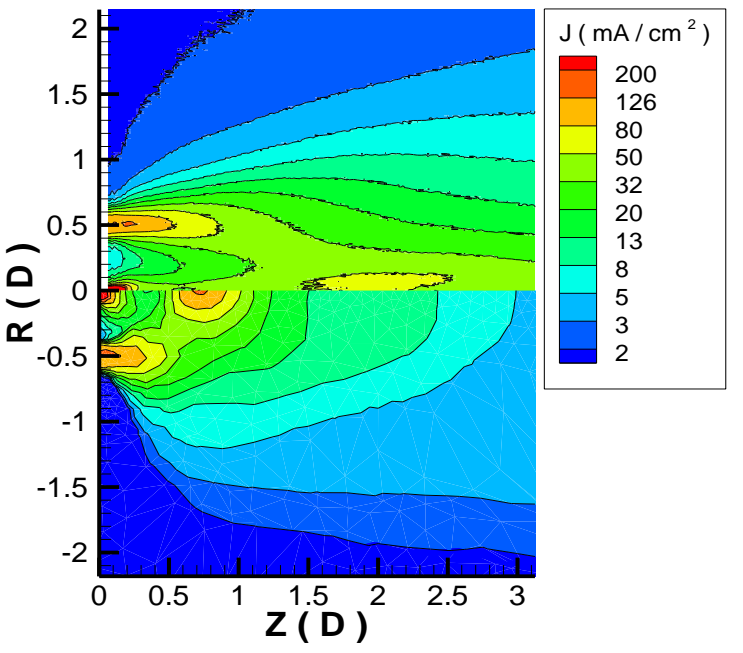

Figure 13: Current density comparison, measured data (top) and constructed input condition (bottom) 


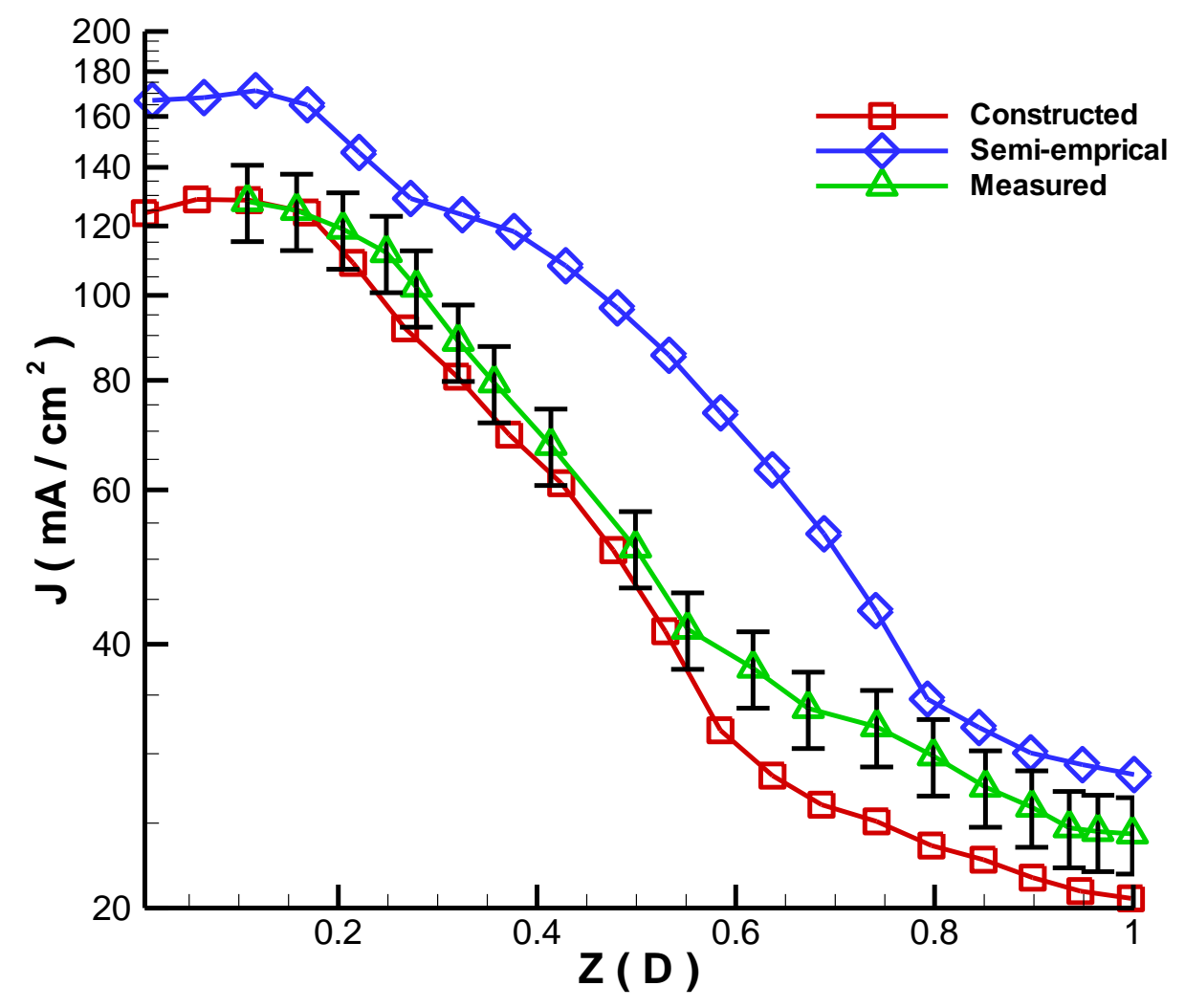

Figure 14: Current density variations along the discharge chamber centerline, measured from the thruster exit (note error bars on measured data are $\pm 10 \%$ )

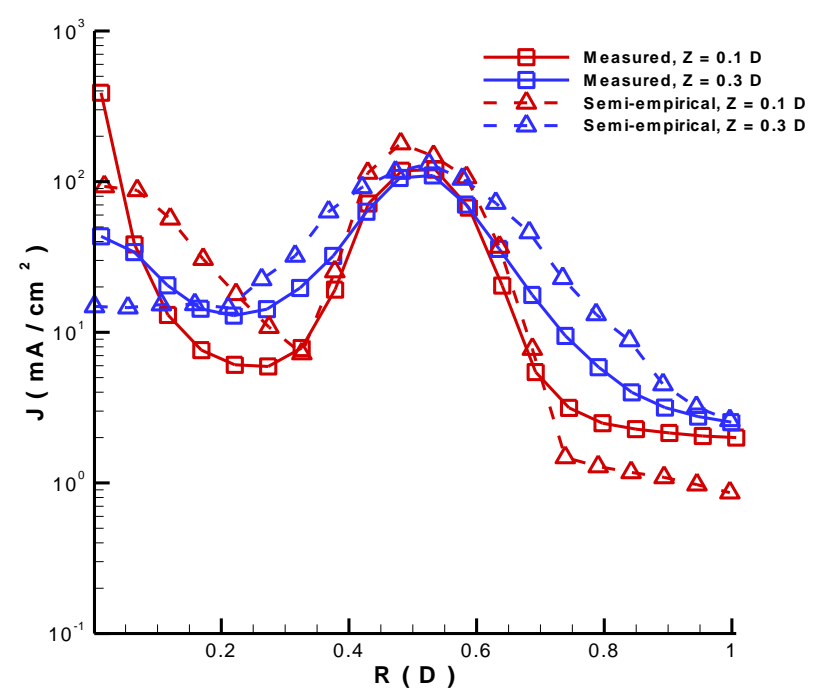

Figure 15: Radial current density comparisons between measurements and predictions via semiempirical method at various axial stations

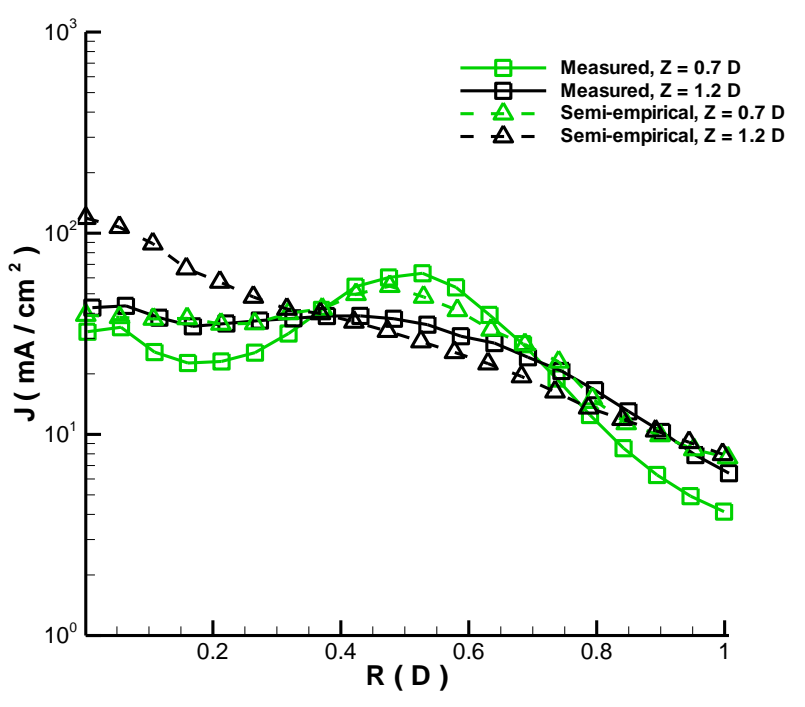

Figure 16: Radial current density comparisons between measurements and predictions via semiempirical method at different axial stations 




Figure 17: Radial current density comparisons between measurements and predictions via constructed method at various axial stations

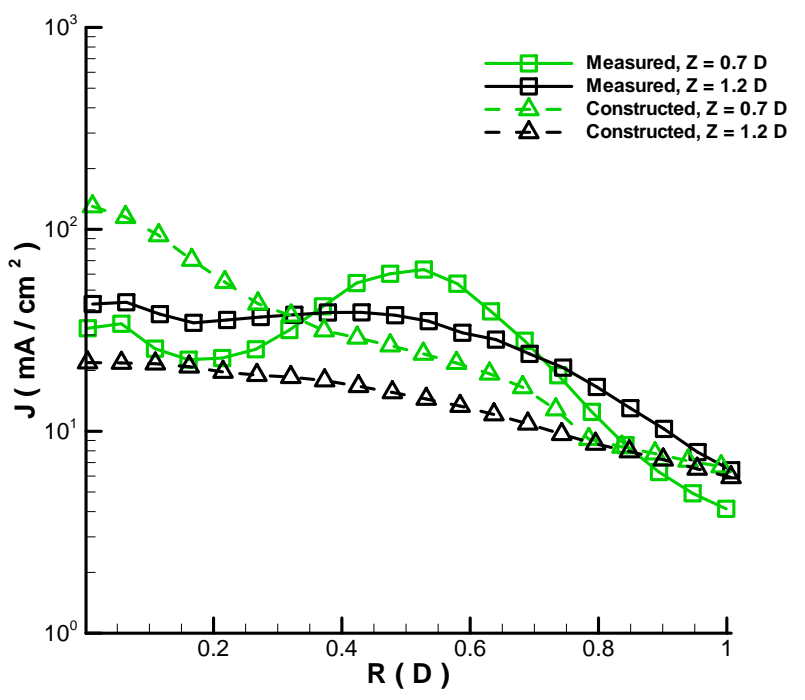

Figure 18: Radial current density comparisons between measurements and predictions via constructed method at different axial stations

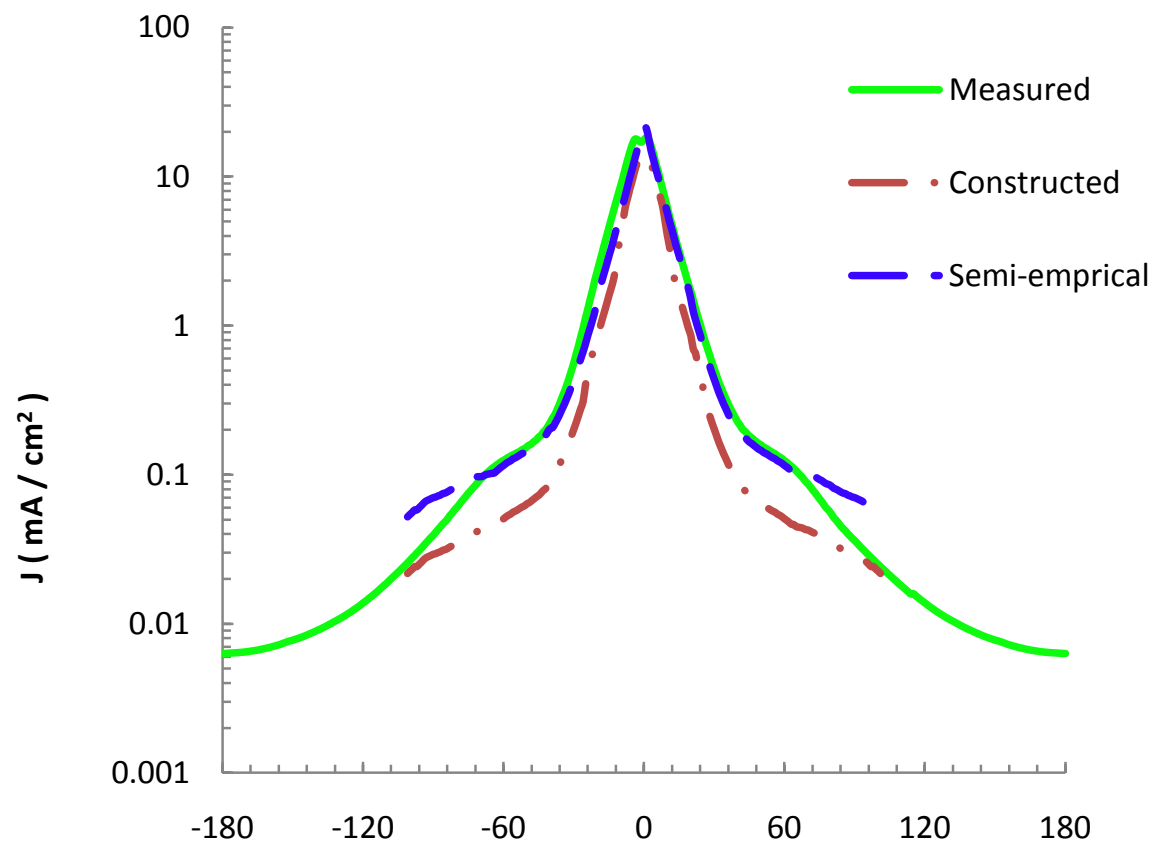

Figure 19: Angularly resolved far-field current density comparisons between measurements and predictions 


\begin{tabular}{|c|c|c|c|}
\hline Method & Measured & Semi-emprical & Constructed \\
\hline $\begin{array}{c}\text { Integrated } \\
\text { Current }\end{array}$ & $21.79 \mathrm{~A}$ & $18.05 \mathrm{~A}$ & $15.98 \mathrm{~A}$ \\
\hline
\end{tabular}

Table 4: Integrated current comparisons

Neither simulation accurately predicts the plume structure outside of the near-field plume region, as seen most clearly in Figure 19. Figure 19 shows significant discrepancies at high angles for both constructed-method predictions and semi-empirical-method predictions. Table 4 shows the integrated currents that are obtained from the far field measurements in Figure 19. These integrations are performed over an identical range of angles for each method from $-100^{\circ}$ to $100^{\circ}$. Each prediction method underestimates the integrated beam current obtained through measurement.

The discrepancies in current density data are due to two major contributing factors. First, the numerical model is limited in requiring fluid-type boundary conditions throughout the entire domain, which are typically not available. For example, Eq. (7) requires a direct value for electron temperature set at the outflow surfaces, but acquisition of electron temperature measurements for such a large range of spatial locations is not feasible. Second, the current numerical model makes strong assumptions about the makeup of the cathode mass flow. Currently, only xenon neutrals are injected from the cathode. On comparing the computational predicted current density at the cathode with the measured current density, it is clear that the cathode flow also contains some xenon ions, as the measured current density is nearly twice that of the predicted current density. The integrated current results could also support this, as the measured integrated current is larger than the discharge current of the thruster, suggesting that the cathode provides a significant far-field contribution to the integrated current. Finally, previous work on cathode modeling also indicates the presence of xenon ions ${ }^{19}$. The current study must assume a cathode mass flow consisting of only neutrals because there is no data available on the ion properties at the cathode outside of current density measurements.

\section{Conclusions}

A general purpose, hybrid DSMC-PIC code has been supplemented with a simulation package, HPHall. HPHall's capability to predict thruster exit conditions has been assessed. The refined conditions more accurately characterize what was previously an area of high uncertainty, as determined in Ref. 2 . The new approach provides a scientific alternative to the semi-empirical estimation of particle properties at the thruster exit by analysis of velocity distribution functions. These refined input conditions for plume simulations enhance simulation accuracy in the near-field plume region, predicting peak current density at the thruster exit to within $1.26 \%$.

One area of future study to better understand the physical situation in the plume is the characterization of the xenon species at the cathode. As has been previously noted, the mass flow at the cathode is made up of xenon neutrals and ions. The current study assumes a cathode mass flow consisting of only neutrals and this aspect requires further investigation.

\section{Acknowledgments}

The authors would like to thank and acknowledge the Air Force Research Laboratory (AFRL) for funding this work through the Michigan/AFRL Center of Excellence in Electric Propulsion (MACEEP). Dr. Mitat Birkan (AFOSR) and Dr. James Haas (AFRL/RZ) are the joint program managers for this center grant (F9550-09-1-0695). 


\section{References}

${ }^{1}$ Boyd, I.D., "Review of Hall Thruster Plume Modeling," Journal of Spacecraft and Rockets, Vol. 38, No. 3, 2001 , pp. $381-387$.

${ }^{2}$ Huismann, T. and Boyd, I.D., "Modeling of Virtual Diagnostics for Hall thruster plumes in a vacuum-chamber," IEPC-2009089, 31 ${ }^{\text {st }}$ International Electric Propulsion Conference, Ann Arbor, MI, 2009.

${ }^{3}$ Bird, G. A., Molecular Gas Dynamics and the Direct Simulation of Gas Flows, Clarendon Press, 1994.

${ }^{4}$ Birdsall, C. K., and Langdon, A. B., Plasma Physics via Computer Simulation, Adam Hilger, U.K., 1991.

${ }^{5}$ Boyd, I.D., and Yim, J.T., "Modeling of the Near Field Plume of a Hall Thruster," Journal of Applied Physics, Vol. 95, No. 9, 2004, pp. 4575-4584.

${ }^{6}$ Reid, B., The Influence of Neutral Flow Rate in the Operation of Hall Thrusters, Ph.D. thesis, University of Michigan, 2009.

${ }^{7}$ Fife, J., Hybrid-PIC Modeling and Electrostatic Probe Survey of Hall Thruster, Ph.D. thesis, Massachusetts Institute of Technology, 1998.

${ }^{8}$ Hofer, R.R., Mikellides, I.G., Katz, I., and Goebel, D.M., "BPT-4000 Hall Thruster Discharge Chamber Erosion Model Comparison with Qualification Life Test Data," IEPC-2007-267, 30 ${ }^{\text {th }}$ International Electric Propulsion Conference, Florence, Italy, 2007a.

${ }^{9}$ Parra, F., Ahedo, E., Fife, J., and Martinez-Sanchez, M., "A two-dimensional hybrid model of the Hall thruster discharge," Journal of Applied Physics, Vol. 100, No. 022304, 2006.

${ }^{10}$ Giuliano, P., and Boyd, I.D., "Spectral analysis of simulated Hall thruster current oscillations," IEPC-2009-084, 31 International Electric Propulsion Conference, Ann Arbor, MI, 2009.

${ }^{11}$ Szabo, J., Fully kinetic numerical modeling of a plasma thruster, Ph.D. thesis, Massachusetts Institute of Technology, 2001.

${ }^{12}$ Gamero-Castano, M. and Katz, I., "Estimation of Hall Thruster Erosion Using HPHall," $29^{\text {th }}$ International Electric Propulsion Conference, Princeton University, Princeton, 2005.

${ }^{13}$ Hofer, R.R., Mikellides, I.G., Katz, I., and Goebel, D.M., "Wall Sheath and Electron Mobility Modeling in Hybrid-PIC Hall Thruster Simulations," 43 ${ }^{\text {rd }}$ AIAA/ASME/ SAE/ASEE Joint Propulsion Conference, Cincinnati, OH, AIAA 2007-5267, 2007b.

${ }^{14}$ Hofer, R.R., et al., "Efficacy of Electron Mobility Models in Hybrid-PIC Hall Thruster Simulations," 44 ${ }^{\text {th }}$ AIAA/ASME/SAE/ASEE Joint Propulsion Conference, Hartford, CT, AIAA 2008-4924, 2008.

${ }^{15}$ Miller, J. S., Pullins, S. H., Levandier, D. J., Chiu, Y., and Dressler, R. A., "Xenon Charge Exchange Cross Sections for Electrostatic Thruster Models," Journal of Applied Physics, Vol. 91, No. 3, 2002, pp. 984-991.

${ }^{16}$ Chen, F.F., Introduction to Plasma Physics and Controlled Fusion, Vol. 1: Plasma Physics, Plenum Press, 1984.

${ }^{17}$ Cai, C., Theoretical and Numerical Studies of Plume Flows in Vacuum Chambers, PhD Thesis, University of Michigan, 2005.

${ }^{18}$ Choi, Y., Keidar, M., Boyd, I.D., "Particle Simulation of Plume Flows from an Anode-layer Hall Thruster," Journal of Propulsion and Power, Vol. 24, No. 3, 2008, pp. 554-561.

${ }^{19}$ Boyd, I.D., and Crofton, M., "Modeling the Plasma Plume of a Hollow Cathode," Journal of Applied Physics, Vol. 95, No. 7, 2004, pp. 3285-3296. 